\title{
Surveillance \& Society \\ Playing at Control: Writing Surveillance in/for Gamified Society
}

\section{Garfield Benjamin}

Solent University, UK

garfield.benjamin@soylent.ac.uk

\begin{abstract}
Gamification has entrenched constant monitoring throughout society. From education to work to shopping, our activities are tracked, our progress is monitored, and rewards are meted out. But this enforced acceptance of constant surveillance constructs a social narrative in which privacy ceases to exist, and the technological tools at work can easily be shifted from reward to control. This is furthered through the shift from a Bentham-Foucault model of power and the threat of surveillance to the actualisation of complete protocological surveillance enabled by cloud computing, data centres, and machine learning. It is no longer the case that anything we do might be surveilled; we can be fairly certain that everything we do probably is being monitored, judged, and recorded. How can we negotiate these changing narratives? Of what fictions do we convince ourselves when we play the "game" called digital society? This article uses the work of Cory Doctorow, Charles Stross, Dave Eggers, and Ernest Cline to assess how fictionality can act as thought experiments for the social conditions of surveillance technologies. Through stories such as Halting State and Walkaway, we explore the collisions between the control-based society of tech companies and the disciplinary structures of traditional states - the points of tension between illusions of freedom, guided game paths, and the exercise of power over users' data and behaviours. The article argues for expanding our perspectives on the reach of game analysis to the broader connected networks of cultural and political systems, to assess ways of responding to the idea that we are being played with, turned into characters in the gamified narratives of control-based surveillance societies.
\end{abstract}

\section{PRESS START \{introduction\}}

The carrot is better than the stick, or so the saying goes. But what happens when the carrot is embedded with tracking devices and is only a score of carrot points kept by a digital gamified system? Marketers would have us believe that nothing increases participation like turning mundane tasks into digitally mediated games. But, while we willingly consent to having our progress monitored in order for the system to know when to dole out rewards, the process also generates large amounts of data on our actions, behaviours, and preferences that can be used for profit or, more importantly, power and control. Computer games have long been a major industry, with roughly 400 million users in the US in 2018 across download, mobile, and online sectors (Statista 2019), so it is perhaps little surprise that ideas from digital game design have been applied to diverse areas such as work, education, health, and consumer behaviour. It is telling that a recent Netflix report considered the online game Fortnite to be a bigger competitor than other streaming services (Netflix 2018: 5). Netflix itself is making a similar move from attention to participation, such as the interactive film Black Mirror: Bandersnatch, which plays with issues of game design, control, and technology throughout, even labelling PAC-Man as "Program and Control Man" trapped in "a fucking nightmare universe, and the worst thing is it's real and we live in it" (Brooker 2018). This sense of being trapped in a maze following pointless game rules and sociotechnical imperatives, endlessly repeating the illusion of choice, is the reflexive theme of the episode commenting on how all our lives are being "played with" by gamified systems and a trend evident in society more widely as well as an undercurrent of game 
studies. For example, Boellstorff (2006: 33) identified three levels at which game studies can approach the role of games in society: game cultures (within individual games), cultures of gaming (between games), and the gaming of cultures (applying game mechanisms to society and culture at large). This approach informs the present discussion in the application of gamification to surveillance, for it is important to assess the role of gamified surveillance within specific systems, as a process of normalisation and control across systems, and at the level of broader power structures at play between entities employing such systems.

This article examines speculative fiction works by Charles Stross and Cory Doctorow, as well as Ernest Cline and Dave Eggers, to discuss gamified surveillance as the underlying logic and power structure of society and its critique within culture. A sociocultural and functional approach to gamification as a power structure will be outlined as a means of analysing the underlying logic of surveillance in society. Beyond technical details and design principles, in the shaping of society, it is our images, writing, and ideas that can define how a system is adopted, integrated, and extrapolated. It is therefore through culture that we must understand, critique, and perhaps even circumvent the trend towards society as a game of total surveillance. In order to enact this critique of gamification and explore potential methods of resistance to the power structures of surveillance, the structure will follow the seven methods offered by Dragona (2014: 239-42) as a means of critique and countergamification for digital society: hypertrophy, exposure of game mechanics, overidentification, obfuscation, desertion, de-gamification, and re-appropriation. These specific foci will be read in turn through specific works of fiction, following the establishing of gamified surveillance societies through their power structures and on to methods of resistance. The discussion will weave between narrative, theory, and society in order to reflect on the critical perspectives made available through fiction as sociotechnical thought experiments. By combining alternative possible worlds with individual character perspectives, fiction can allow us to address both the societal structures of power and the affective experiences of the individual. In the process, gamification is revealed not only as the social narrative that justifies mass surveillance, but the defining structure of control in digital society.

\section{MENU \{existing literature\}}

Gamification is now an established practice and has received increasing attention from academia, including in the particular case of surveillance (Whitson and Simon 2014; O'Donnell 2014; Cybulski 2014; Andrew 2015). Definitions and value judgements of gamification are many and various. While the specifics of the term tend to differ based on whether the writers work in systems design (Deterding et al. 2011), psychology (Oravec 2015), game design and games studies (Bogost 2015), or any number of other fields, all focus on extracting some (never all) aspects of digital games and applying them to other areas to increase participation and data collection. It is this partiality that forms the basis of a critical view of gamification by games scholars such as Bogost for its extrinsic reward system and appropriation of play for market gain. Gamification creates only the semblance of play, trivialising games as a means to control rather than treating play as a serious and valuable end in itself. Indeed, computer games themselves were an early target of gamification, beginning most notably with Microsoft's introduction of "achievements" as "a system of ingame surveillance" (Cybulski 2014: 427), shifting the emphasis from individual games to platforms such as Xbox Live. In order to reach all three of Boellstorff's levels of games (and gamification), therefore, the technical specificities of game design are less important to the present discussion than the social impact of the phenomenon in terms of surveillance. To this end we will examine the embedding of digital games and gamified surveillance in culture, as seen through literature. If, in the process of gamification, "the fiction, ambiguity and uncertainty found in games are also purposefully absent" (Dragona 2014: 229), then we will reinsert this productively antagonistic role of fictionality by examining literature and its relation to society. Speculative fiction in particular is not only echoed in the game world as an alternative setting - included too in gamification as the process of extracting behaviours and power structures and displacing them into the abstracted (fictionalised) realm of scores and rewards - but also acts as a thought experiment for the social implications of technology, potential responses by humans, and alternative modes of organising society against and outside existing power relations. 
Two key concepts that frame current debates in the interplay of surveillance, power, and society are platform capitalism (Srnicek 2017) and surveillance capitalism (Zuboff 2019). Srnicek (2017: 6-13) situates digital platforms in the economic history of capitalism, defined by constant growth and constant technological change in which workers are deskilled, made pliable and interchangeable. This highlights the trivialisation of contemporary work and life and sets the scene for gamification to promote engagement, productivity, and growth. A platform-based system uses data as a basic resource, bringing people together in a contrived social environment focused on building networks with constant user engagement. Zuboff (2019: 181) treats the processes of surveillance itself as the defining feature of current digital society, bringing together a series of themes that had been emerging in digital cultural studies and culminating in behaviour modification as the most dangerous use of technology in networked society. This echoes work on gamification power structures that "game" society itself, as "a technique for behaviour regulation ... via codified positivefeedback mechanisms" which "aims at the regulation of behaviour while circumventing attitudes" (Schrape 2014: 33, 43). Gamification plays a different game of power, converting cultural attitudes into measurable behaviours that can be monitored and manipulated without ostensibly affecting the underlying psychosocial or ideological operation of society. Zuboff (2019) is instructive in her direct discussion of gamification, specifically of Pokémon Go! as a combined data harvesting and behaviour modification tool, converting play into a market-serving phenomenon across digital and physical spaces, inside and outside the game. She usefully highlights the higher order game of society that is at play within, through, and between gamified systems. However, here as throughout the text, she overemphasises the newness of her framework, which is a culmination of trends in technology and its critique in thought, failing to correctly acknowledge existing literature and the longer impact of gamification on work, health care, education, and other sectors. Whilst critiquing the "unprecedented asymmetries in knowledge and the power that accrues to knowledge" through which surveillance capitalism operates, Zuboff $(2019: 148,362,334)$ holds a less than critical stance towards capitalism in general, placing surveillance as a "rogue" form of the system even as she moves past platforms to tackle the underlying logic which appears to itself be rooted in capitalism.

Unlike Zuboff, Srnicek emphasises a continuation of mainstream capitalism onto platforms and is critical of capitalism as a system already based on asymmetries and inequalities of power. But, like Zuboff, his focus on the economic imperative does not go far enough into the informational imperative that defines the underlying power structures. Zuboff (2019: 306) rightly emphasises surveillance power as emerging out of but separate from the technologies themselves, but there is more at stake than simply the pre-existence of technology. While any given technology might have positive and negative uses for different people, it is not created neutral, and Zuboff misses the underlying power structure over informational reality that was at the heart of (for example) Google's search algorithm. Outside economic application, the research underpinning this shift in technology was essentially the gamification of the web as a social construct: it became the relational scores of backlinks (and increasingly other usability scores such as accessibility across devices) from which the rankings were derived. Surveillance capitalism itself emerges according to the logic of gamified power through technology, a combination of data collection and scores or rewards where power is measured by money, although it is anything but playful. Digital society is founded on the creation of a gamified power structure to control the flow of information, in which the objective is clicks and views, and the aim is complete and compulsory participation.

Andrejevic (2009: 52, 61, 53) previously identified a lack of choice over participation, in that the premise of the exchangeable value of data "requires willing submission" by users and/or consumers in order to gain access to exchange of material and social value. Even after paying this entry price, we are funnelled into active participation in systems beyond our control. This exploitative process embeds users within "enclosures" as interactive spaces and processes of a "monitoring embrace" of virtual(ised) space. This resonates with game worlds as total surveillance-control systems and smart cities as highly surveilled public spaces; the process of enclosure is the conversion of digital, physical, and social realities into surveillance games. Clarke (1988: 499-511) prefigured these shifts when he defined dataveillance as "the systematic use of personal data systems in the investigation or monitoring of the actions or communications of one or more persons." He outlined techniques including the integration and concentration of data, the screening, validation and profiling of individuals, and cross-system enforcement and identified individual, collective, 
and societal risks of dataveillance, including many of the current debates around selective advertising, bias, and errors, and asymmetries in knowledge and power. Clarke also rightly warns against centralisation, attempting to replace it with a "law of requisite variety for information systems," but in doing so failed to anticipate the prevalence of standards, third-party data sharing, the massive scale of data collection and use, the rise and value of inferred data, and the quasi-monopolies that can design whole systems to be integrated (e.g., Google). The focus here is therefore not surveillance capitalism but surveillance governance. Governance, as the establishing of systems of rule and control, is separate from government, which tends towards the specificity of nation states no longer suited to globalised digital society. Governance today manifests in a combination of states, corporations, platforms, personalities (celebrities, thinkers, and "influencers"), and design-social norms. Surveillance governance is a protocological power structure under which access-control must always be monitored and gamified, but the control structure is distributed, a general logic embodied across many entities. Gamification is the tool for this form of governance enabled by surveillance.

The literary texts used in this article lie in what I have previously described as the "third wave" of cyberpunk (Benjamin 2016). Rather than hacking technical (Gibson 1984) or linguistic (Stephenson 2011) systems, in the work of Cline, Stross, and Doctorow (as well as contemporary media such as Black Mirror) focus is placed on finding exploits in cultural and social systems, often using established technoculture to exert influence and control or to break down corrupted systems and build alternative societies. This echoes the move in surveillance theory from Foucault's disciplinary power to Deleuze's (1992) society of control and on to its distributed fulfilment in Galloway's (2004) "protocological" system, which programs and controls our behaviours. A discussion of gamification is therefore informed by surveillance and society in terms of the "exploit" (Galloway and Thacker 2007: 101). What the control society converted from stratagem to security and exception, the protocological mobilises through gaming and inception. This framing establishes a ground from which we can examine the third level of game studies - gaming of cultures - and assess the impact of game protocols (without game play) as a system filled with exploits for use by those with power and those trying to counter it. We can also use from gaming the concept of cheating, applying it to the rules of society at large. For example, Consalvo's (2007: 113) four key types of cheating, defined broadly as using additional knowledge to gain an advantage, can be equally applied to surveillance:

- taking advantage of glitches - finding the gaps in law and social contracts that allow surveillance

- taking advantage of people - exploiting consent and the use of gamified scores to enhance social standing

- taking advantage of code-embedding automated mass data collection in all systems, unfair algorithmic decision-making based on this data

- taking advantage of third-party systems - transferring or selling data, statecorporate agreements, gaming social systems

These processes can be seen in, for example, China's "social credit" system: making use of a fusion of state power and existing corporate data platforms combined with incentives for citizens to embed gamified surveillance into society as a whole through unified "scores" that can have real impact on financial and other aspects of people's lives. But this blatant example of gamified surveillance and state power-which has been foreshadowed in several of the works discussed in this article-is only one obvious implementation. As Whitson (2013: 175) describes, "data collection is achieved by trying to trick the worker into thinking they are playing when they are not" and often for no other purpose than entrenching the will to participate in widening social contexts. For example, in Stephenson's (2011: 135-36) Reamde, a fantasy MMORPG (massively multiplayer online role-playing game) is used as a host for gamified work even though the automated system that converts real world data of people moving through airport security into goblins and dungeons could remove the need for humans altogether. The economic and social impact of participation is its own reward, furthering the reach of game and gamified systems and entrenching the power and influence the owners of these platforms hold. 
Digital games and surveillance share a military history and a concern with behaviour and have merged again in recent times in the Snowden-leaked documents, which included discussion of the NSA's potential for games. The "military-entertainment complex" (Crogan 2011) therefore receives a new instantiation in gamified surveillance (Whitson and Simon 2014: 311). Games were seen by the National Security Agency (NSA) and Government Communication Headquarters (GCHQ) as a risk - a possible secret communication channel for terrorists - but also an opportunity. As Wang, Haines, and Tucker (2011:31) suggest, "advanced cybercommunities are communities in which perfect surveillance is possible-software tools allow everything to be observed, recorded, archived, pored over at a later date and acted upon." The shift of more and more of our lives online, not just as an area of alternative activity in new cybercommunities but the digitalisation of "real life" from the workplace to our social interactions, places increasing amounts of data into the hands of the corporations that enable the platforms we use but also into the hands of the states that allow, support, or exploit these platforms. Whitson $(2015: 341,339)$ labels "gamification as a technology of government" (though we might properly say of governance), which functions by "enrolling individuals in normalization projects." For Whitson, it is less about metrics and data collection than it is about the feedback such data enables. By giving rapid personalised feedback, users measure themselves against the codified objectives of the "game" in a process that shapes and reshapes the approved paths that "players" might follow. Gamification is therefore a "tool for Foucauldian biopolitics," and gamification techniques "could indeed be harbingers of a novel style of governance" (Schrape 2014: 37). This emerges in postFoucauldian, Deleuzian control, and protocological systems. Speculative fiction allows us to stage the implications of these methods of governance, to highlight warnings of their potential exploitation, and to develop collective responses.

\section{INSERT \{hypertrophy: "Scroogled"\}}

Doctorow, famed privacy advocate, author, and blogger, often deals with the crossover of corporate and state surveillance. Where Stephenson's Reamde shifts airport security into an online fantasy world, in Doctorow's ([2007] 2015) short story "Scroogled" it is raw data that is transferred from Google as part of the corporate takeover of border control. The fusion of state and tech giant leads quickly to a system of total surveillance, embracing privatisation not simply for efficiency but for its existing large user base and Google's tendency to manufacture participation. There is a social cheating going on here, gaming the system by exploiting a legal loophole. While simply handing over a user's search data might be legally questionable, shifting the surveillance onto metadata removes such qualms (as least as far as state and corporate ethics are concerned). By showing border control agents which ads a traveller has been shown by Google, their search history is implied as an indicator of crassly aggregated search terms. This can then be used as leverage (or an excuse) for more intensive privacy invasion and justification for revealing full personal data, activity, and search histories. In the story, the system is predictably and almost humorously error prone-the protagonist gets stopped due to having been served rocket kit ads after searching for the "launch" of a new coffee called "rocket fuel." While a user may harmlessly ignore such an ad at the top of a Google search, Homeland Security is less forgiving, and the real world escalation is clear.

An interesting counterpoint to the integration of tech and state in practice is seen later in speculation on the internal workings of Google as its culture shifts into national security contexts. The invasive surveillance of search histories leads to stark divisions of cleared and suspected travellers and employees - compliance feeds off the tech culture FOMO (fear of missing out) and the will to participate, with the internal higher status of "cleared" employees leading to new inequalities based on transparency (for more on this, and the gamified drive to participation, see the discussion of The Circle below). While there is no explicit gamification here - the games of power occur behind the scenes between state and corporate interests with users caught in the crossfire or treated as tradeable items - the state uses its surveillance powers and control over access to projects and abilities (to travel without harassment, for example) to gamify compliance and close down digital technologies as a platform for "deviant" behaviour. This has been played out in the Chinese social credit system, which has been used to deny travel tickets (rail or air) to twenty-three million citizens based on "social offenses" (Kuo 2019). Similarly, the crossover of state border control and 
corporately owned social media is occurring already in the United States ESTA (visa waiver) program, which asks users (optionally, for now) to provide details of their social media accounts for enhanced background checks.

Dragona (2014: 235) states that "gamification assists in narrowing identity to identification" and that it is undertaken willingly. Our use of technology reduces our identity to our measurable behaviours, which are easily surveilled. This process reflects Deleuzean "dividuation," breaking individuals into data parts that can be reformed into new functional assemblages. Whitson (2015: 343) states that "dividuals are then governed automatically through databases and levels of access and exclusion." We see the move from identity to codes and passwords, from discipline to access control, and what Andrew (2015: 361) describes as the shift towards device ID as a replacement for personal identification and means of tracking individuals through behaviours and interfaces. Combined with physical location tracking, these aggregates of controlbased methods can be used for manipulation and discrimination to perpetuate inequalities of power, what Galloway and Thacker (2007: 40) identify as new forms of control-based sovereignty that shift from directly manipulating individuals-nodes to exploiting the flows of networks/topologies. Gamification is therefore the fulfilment of what Deleuze critiqued as the society of control, manifested in "Scroogled" as a fusion of disciplinary state measures with corporate distributed methods of protocological control that quietly restricts behaviour even as it offers new modes of participation.

"Scroogled" also therefore demonstrates the tendency towards function creep that has in part enabled the ubiquity of surveillance across many digital platforms. Whitson (2015: 351) describes how "surplus value is created from the information we trail along behind us, information that is then used to govern our consumption habits," and it is here that the feedback mechanisms of gamified systems reveal their hunger for data and increased surveillance into black box systems of control. O'Donnell (2014: 350) describes how for in-game surveillance, "the opacity of these systems was no different than the opacity of a game's underlying rules and systems." Perhaps it is this embedding of surveillance and governance into alreadyopaque systems that has aided its creeping influence. Whitson (2015: 342) describes how a mix of administrative governance and self-governance has emerged to replace the breakdown of the panopticon in the face of individualism and neoliberalism. Scroogled's focus on privatisation of total surveillance expresses this shift. Gamified systems further this trend with an inversion of the panopticon; we do not know how or where it works, but we do know (or at least assume) that it is there all the time watching, monitoring, and manipulating. We don't know the rules of the game or sometimes even which game(s) we are playing at a given time. Our whole reality becomes a will to participate without knowledge of the nature, rules, or implications of the broader games of society we are manipulated into playing.

\section{PRTSCR \{exposure of game mechanics: Halting State\}}

A blurring of state and game, of rules and implications, can be seen in Stross's (2008) Halting State. Aside from the narrative itself, this book is notable to the present discussion for two key reasons. The first is that it employs a second-person narrative that echoes game-like paths akin to choose-your-own-adventure stories, guiding the reader through a false sense of choice to a limited set of outcomes. With the singular narrative of this book, however, the second-person perspective also evokes a sense of surveillance. It is YOU who are doing these things. We know you are doing them, we are recording you doing them, and we know in advance that you have no choice but to do them. This second-person focus resonates with Chun's (2016: 3) assertion that new media is "nYOU media," that technology is a habitual "function of you." Stross's framing of the narrative in this way emphasises the personal and functional monitoring of the system, not the agency of the characters or the reader. This reflects the state of the main characters, gradually swept along into a deadly game that spans digital and physical realities, with shadowy controllers and opaque rules but high stakes. The gamification moves beyond a specific platform or even the functional design of games, blurring again the line between state and game technology. As one character remarks on the gamified police monitoring and informational technologies, "CopSpace? That's not a game, that's a metaverse" (Stross 2008: 55, 160, 241-42). The novel is set to a backdrop of an in-game heist, an attack on a fantasy world bank that serves as a locus for trades between different game systems and third-party value 
systems. By the end of the story, however, the characters have become embroiled in an augmented reality spy game ("SPOOKS"), which is being used as a cover for genuine state and corporate espionage. The game itself is described as "almost tediously realistic" but that "rumour has it that the first SPOOKS campaign got the beta-testers arrested and questioned for a week under the Terrorism Act before the police realized it was a game." In the paranoid age of networked systems and the war on terror, there is little suspension of disbelief required here. While surveillance has traditionally been the purview of the state as an extension of the monopoly on the legitimate (or legitimated) use of force, here we see a reflection of the processes at work in the digital surveillance society to translate these functions to private companies and thereby extend state and capitalist reach. SPOOKS takes it further by distributing the process to willing volunteer citizens who take on small unknown acts of surveillance to progress within the "game." This process is described as "the biggest renaissance in HUMINT-HUMan INTelligence-since the Cold War. It's all mediated through artificial reality and live-action role-playing games like SPOOKS ... Would you believe it used to cost us ten thousand euros a day to put a full surveillance team on a suspect? Now we've got volunteers who'll pay us to let them do our leg work!"

The gamification process applied to the act of surveillance itself spills over from the digital mediation of games into physical reality. In describing the implications of this, we see a hint of Stross's (2008: 219) style of eldritch writing, combining computing with horror (in the manner of his The Laundry Files): "looming at the edge of the universe is a thing of horror. The games have imploded into reality." The unknowable horror in Halting State is the surveillance-gamification system as a whole, the military-entertainment complex hiding in the code that underpins our digitally mediated reality. The story expresses the military discourse whereby "players become pawns in larger games of states and terrorists" (Whitson and Simon 2014: 312). The opacity here calls into question the nature of participation in gamified systems. If we do not know the full extent or nature of the rules we are supposed to follow, we become at best willing but unwitting participants. As Stross (2008: 257) writes, “you're playing a game you don't understand the rules of against an artificial reality engine." This leads towards "gamification as a symptom for an emerging new mode of governmentality" (Schrape 2014: 21), which perhaps hews a little more closely to our present situation than we would care to admit.

The second key point of interest for Halting State is that Stross was unable to finish the trilogy. Following a sequel-Rule 34, focusing on spam, porn, murder, and networks of organised crime-Stross felt compelled to stop writing the series. As he wrote in a blog post, "Halting State wasn't intended to be predictive when I started writing it in 2006... The science fictional universe of Halting State and Rule 34 is teetering on the edge of turning into reality .... The Snowden revelations have systematically trashed all my ideas for the third book" (Stross 2013). Stross's speculation was no such endeavour. While attempting to write near-future fiction detached from our reality, as a warning and exploration of potential misuses of surveillance and game technologies, he detailed processes that were already underway. In Halting State, the developers "cheat" (following Consalvo's list detailed above) with the end-user agreement, employing variable terms and conditions (an all too common practice in real world agreements with pages of legalese that nobody reads before clicking agree) to convert SPOOKS into a "click-through" to the official secrets act giving consent for background checks to be undertaken (Stross 2008: 220). Stross was unintentionally channelling the real world connections between surveillance and games, at least in the imagination of the security services and defence contractors, and the conversion of games into something altogether different. And yet, despite Stross's move away from this inadvertent realism, it is precisely through pushing technological realities to extreme ends that fiction can effect an exposure of the underlying systems of power and control in which they are embedded and perhaps (as we see across these texts) create spaces in which we can speculate on methods of countergamification. Perhaps underpinning Stross's reluctance to continue was the difficulty in effecting a speculative distance in which his fiction could operate in critical estrangement. Without such distance, it is possible that fiction becomes too descriptive, losing its ability to "play" with the problems of our current reality. 


\section{ALT \{overidentification: The Circle\}}

In relation to the problematic appropriation of play in gamification, Whitson (2015: 346) writes of "feedback loops, not fun," stating that there is "often no 'game' in gamification," referring to Bogost's (2015) critique of gamification as "bullshit," but instead asserting that a lack of fun does not mean gamification is a failure. Rather, the removal of fun signifies a shift in focus towards (positive) feedback and reinforcement. Here the act of exposing the game mechanics as explicit scores (whether social, health, or in the workplace) actually normalises the game control system, forcing users to identify with the desires and objectives of the system. O'Donnell (2014: 357) echoes this move away from a privileged position of games as a special medium, suggesting that instead "these new 'gamed' experiences enter into new dialogs with existing forms of power and structure." Games are embedded in a history of sociotechnical development, and we should therefore not see quantification, feedback, and control as one functional expression of games. The influence runs the other way around. Gamification is a logic of control in society, the protocological management of citizens in developing compliant behaviours driven towards objectives delivered by anonymous systems. The history of gamification within games feeds back into the surveillance by the NSA and GCHQ of Xbox Live, World of Warcraft, and Second Life (Ball 2013), echoing Stross's concerns that fiction was already reality. Following the Snowden revelations, a new form of speculative fiction was required to address the power structures at work in a gamified surveillance society and to provide the basis for discussing alternative ways of organising access to information and redefining systems of control.

Counter to the history of games as military objects, videogame culture has its roots in arcades as performative social spaces with scoreboards (Cybulski 2014: 419), carried through into the participatory element of gamification based on the "constitutive social and experiential dimensions of games" (Deterding et al. 2011: 13). In their broadest sense, touching on definitions of virtual worlds and cybercommunities more generally, "'games' are human systems of rule setting, enforcement mechanisms, and emergent processes, along with related forms of rule-following and role-taking behavior" (Oravec 2015: 1-2). As a method of forming social systems, gamification is therefore "a drive to extend in-game governance measures outward" (Whitson and Simon 2014 313). It exists, however, largely as part of state and corporate imaginaries, for while "gamers are ordered, disciplined, and knowable subjects," we find that "players, more often than not, tend to be more unruly than the rules of their games would presume them to be" (Ibid.). This echoes Galloway and Thacker's (2007) exploit and Consalvo's (2007) cheating, a counter-behavioural trend in gaming whereby it is the rules themselves with which we are playing. Gamification is therefore a futile attempt to make behaviour "safe and predictable" (Bogost 2015: 67). But, as we have discussed above, by bypassing attitudes and focusing solely on behaviour, the processes of abstraction that convert messy and chaotic human life into measurable and rewardable metrics is itself a political gesture. Gamification "holds the potential to transform the very social space itself" (Schrape 2014: 28). The success of feedback loops through scores and increased status manufacture a willingness to participate in these new systems with opaque rules and ever-hungrier surveillance mechanisms.

Eggers's novel The Circle is not particularly prophetic. It is not particularly speculative. Rather, it could perhaps best be described as the amalgamated five-year plans of Google, Facebook, Amazon, and a handful of other tech companies and billionaires - a convergence of what is currently still disparate into a homogenous force for social disruption. It is more a tale of monopoly and technology supported business ideology than it is of science fiction. Margaret Atwood (2013) argues that it is not even dystopia, for "there's no sadistic slave-whipping tyranny on view in this imaginary America.... Instead we are in the green and pleasant land of a satirical utopia for our times." At base, The Circle is an expression of very present-day drives towards total surveillance, total privatisation (and monopolisation), and total participation. But, counter to Zuboff's (2019) emphasis, profit does not feature strongly in the rhetoric of the eponymous corporation. The focus here is on the accumulation of power, through data and control, gaming the system of society in the broadest sense rather than playing the specific game of capitalism. The Circle is therefore an embodiment of gamification par excellence. The setting for the narrative is the removal of anonymity and the ability to opt-out from online services, the takeover of all systems and platforms (eventually even government and democracy) so you have no choice but to use The Circle, and you cannot use their products 
without linking it to your singular actual identity. This is augmented by a hyperactive version of the Silicon Valley corporate mentality, exemplified in the internal "Participation Rank, PartiRank for short. Some people here call it the Popularity Rank, but it's not really that. It's just an algorithm-generated number that takes into account all your activity in the InnerCircle" (Eggers 2013: 100). Work is here transformed not only into gamification for the actual tasks of employment - such as constant monitoring of customer feedback scores - but gamification of the level of participation in the company as a social entity. Employees' personal lives are gradually consumed by the need to move up the various different ranking systems, seeking approval in an increasing number of corporately enforced social games.

The corporate level function creep, from searches to social media to society, echoes trends in real tech giants. Google's use of the online gig economy for its controversial defence contract named Project Maven (Fang 2019) echoes Reamde's gamified privatisation and distribution of traditionally state-controlled functions. But Google's example operates almost like terrorist cells, with the compartmentalisation of microtasks meaning that many workers were not even aware of who they were working for or what they were working on. Distributed systems are used within a hierarchical structure to maintain control. Given the backlash by Google employees to knowledge of the Pentagon contract, the outsourcing could also have been a move to specifically avoid using internal staff. The process converted citizen science and participatory techniques-used throughout The Circle-into methods of manipulation, gaming the system to simultaneously play roles of innocent neutral tech company and government contractor. This echoes Stross's SPOOKS enacted by the neighbourhood watch of the future. The Circle's vision for total transparency and collective surveillance is enacted today in the Amazon home security app, digitising neighbourhood watch to increase participation but in doing so - and in true algorithmic style - amplifying existing (e.g., racial) bias. The problematic nature of "turning everyone into cops" (Haskins 2019) quickly devolves into collective paranoia, revealing the underlying economic motive of selling more internet-enabled security cameras. The gamification and participation processes at work mean that "in the world of crowdsourced surveillance, 'Big Brother is us"” (Schafer 2013: 434). As Eggers (2013: 385, 386) writes, following the examples of Google, Facebook, Twitter, and others, "The Circle has a knack for getting people to participate" with the aim that "with full participation comes full knowledge." The distribution of surveillance uses supposed transparency to embed social self-measurement and self-control. In a stylistic sense, and echoing the shift from changing attitudes to controlling behaviours, the openness of the protagonist's inner thoughts to the reader contrasts starkly with the necessary performativity of her outwards transparency within the narrative.

But hiding (or not) within this veneer of transparency - following Proudhonian or Orwellian maxims with "Secrets are lies // sharing is caring // privacy is theft" (Eggers 2013: 303)-is the drive towards a new social order, one of certainty powered by corporate technological advances. The belief here is that large scale data collection (otherwise known as mass surveillance) and gamification can be applied to eliminate all problems - from homelessness to Congress (Eggers 2013: 370, 391). The drive to participation and supposedly open ideology conceals a political and economic agenda that seeks nothing more than to subsume all of reality into the corporate data centres. Nobody, not even the homeless, not even the political elite, are outside of this gamified surveillance system. This is the pursuit of power in its purest. As Bogost (2015) would say, the ultimate pursuit of gamification is only ever more gamification. Enabled by surveillance, gamification takes on a homogenising approach to individual behaviour and overarching social structures, setting up the self-perpetuation of gamified surveillance as a new political system.

\section{ESC + SPACE \{desertion and de-gamification: Walkaway\}}

The collision of social orders and differing uses of technology is the topic of Doctorow's 2017 novel Walkaway. The plot concerns the mechanisms of control in "default" society, controlled by super rich "zottas," and the attempts by various "walkaways" to establish alternative ways of living using technology without the exploitative structures. The derogatory emphasis of the term "default" used by walkaways interestingly echoes its use as a "noob"-equivalent insult on games such as Fortnite, where they refer to the default appearance of users who have not invested the time, skill, or money to improve their digital identity. 
Walkaway follows Doctorow's 2009 novel Makers with its focus on accessibility of 3-D printing technologies and open source designs but extrapolates the speculative element into other areas, up to and including uploaded consciousness running on distributed computer systems in space-the ultimate "walkaway" from restricted default society. Underpinning the issues is the changing nature of power relations and how the apparently distributed neoliberal (or platform-surveillance capitalist) system copes with those who simply refuse to participate. As Doctorow (2017: 370) writes in Walkaway, the real motivator is "power. Money's just keeping score." This is a sentiment played out in his first novel—Down and Out in the Magic Kingdom - in which the whole of a post-scarcity economy transitions into a gamified social network. In this earlier work, money is replaced by Whuffie as representative of the "true essence of money ... your personal capital with your friends and neighbors" (Doctorow 2003: 113). Gamified power is linked to an arbitrary score. All that matters is the visibility of this score and its link to social status supported by participation. We can query an individual's Whuffie just as in real life we can check how many "likes," "followers," or "friends" they have on social media or see their other displays of wealth and power. Society has always developed elaborate mechanisms to act as a social leaderboard, as well as methods of excluding those who fall below the required status for a given situation, but in digital society these are tracked automatically and displayed clearly without the need for subtle social cues. Dragona (2014: 243) suggests that "gamification is the latest and most sophisticated strategy of the vectoral class," but is it really that sophisticated? In the context of reducing gamification to social score-keeping, it is if anything a simplification of asserting dominance. By abstracting social standing to trackable and controllable metrics, it simply displaces the complexity of human social life into small and clearly defined models. Even multigamification (Oravec 2015) can be seen simply as a game between these different games, measured by number of participants. The difficulty instead lies in imagining alternatives to such compulsive scorekeeping.

This quest for an alternative is Doctorow's aim in Walkaway, and even in the eponymous dissenters there is the risk of a return to gamified control. As one character muses, "in a gift economy, you gave without keeping score, because keeping score implied an expectation of reward," but "in practice, it was so easy to keep score, the leaderboard was so satisfying that she couldn't help herself" (Doctorow 2017: 43). The difference here is the lack of link to social standing or power, compared to those who wish to impose a game-based leaderboard as a concrete measure of influence - gamification as a stateless political system. This would be a society ruled in practice by "influencers" and generic "high achievers"- those who could "game the system" in order to gain the highest scores. Doctorow (2017: 84) bemoans these approaches, his characters complaining about the number of "crashed projects where gamification had run wild, so financialized that every incentive distorted into titanic frauds that literally left structures in ruins." Gamification here is pure competition, a combination of militarism and toxic masculinity. This highlights the short-term focus on productivity in gamified systems, leaving participants ultimately unfulfilled and doing subpar work. One character instead suggests that "if you build systems that make people focus on mastery, cooperation and better work, we'll have a beautiful [society] full of happy people working together well" (Doctorow 2017: 83). We still see a combination of individual and collective aims, but it is inverted. Competitive individual productivity through the social drive of rankings is replaced by cooperative productivity for the benefit of all supported by individual motivation to improve. The system of surveillance capitalism rears its ugly head again, as it is the competitive nature of the power structures that enables gamification to succeed as a model for society.

Doctorow ties this back to surveillance with some interesting observations. Not only are the walkaways largely unconcerned with their open source and accessible information being monitored by "default," but the contradictory status of the super-rich in relation to surveillance is also highlighted. In Doctorow's (2017: 170) fictional world, "Zottas do surveillance to themselves. It's not done to them .... The eyes and ears are recording angels that remember everything forever. They're choices." This is a system borne out in reality, with security details and protective monitoring for wealthy and powerful individuals (and their subjectified children being raised in such a normalised surveillant environment). For the subjugated masses, gamification is to enforce compliance and create a perpetual drive towards competitive productivity. For the super-rich it is minimising risk (theft, assault, health, etc.), linked to the ultimate risk of mortality and 
the loss of power. In Walkaway as in the longevity projects of Jeff Bezos, Elon Musk, and Google's Moonshot projects, the super-rich are already on their way to immortality, which will inevitably remain unevenly distributed. Where willingness to participate is manufactured among citizens, combining a fear of missing out with the need to rise in social status, the willing participation by the ruling elites themselves acts as the performative participation in their elite status. Our society is already prepped for surveillancebased mass gamification, providing clear pathways to improving our individual scores and thereby feeding into the myth that anyone can rise in wealth, power, and influence.

\section{SHIFT \{obfuscation: Ready Player One\}}

The question of the agency of the individual against established corporate power is played out in Cline's (2012) Ready Player One. Set in a highly unequal future, in which the physical world has degraded severely, but an online platform "the OASIS" offers opportunities for anyone to better themselves. When the platform's owner dies, the succession of the company is turned into a global competition - an Easter egg hunt "game" based on videogame culture knowledge and the ability to play retro games. The protagonist, Wade, is a "gunter" attempting to win this game, countered by the main antagonists in the form of the OASIS's rival company IOI trying to hack the culture and game the system in order to take control. Much of Ready Player One takes place within the online world. In such virtual environments, there is "absolutely no privacy" for avatars (Wang, Haines, and Tucker 2011: 39), a persistent problem throughout the novel given the high stakes of the "game." Anonymity within the OASIS is undermined by school records (for the highly popular in-world schools providing far greater access to education than the dystopian physical world). The gamification of education and the integration of school systems with online worlds exposes privacy leaks and security vulnerabilities. This is a reflection of the rise in calls for data gathering and even facial recognition in schools today, all powered by third party corporate entities. In Ready Player One, IOI uses this to physically locate and attempt to murder the protagonist - "it was a stupid mistake, but I'd enrolled the year before the contest even began. Before I became a gunter. Before I learned to conceal my real-world identity" (Cline 2012: 143). Education in the online world did not go hand in hand with education about the online world, a real life problem for actual users of digital technology. In virtual environments, even other users can surveil avatars to high levels. As the protagonist describes of IOI, "they had collected thousands of screenshots and vidcaps of my avatar over the past year" (Cline 2012: 291). The use of this inefficient method of surveillance, usually only employed by casual observers or those without access to the system itself, highlights the power inequalities between platforms, particularly when intruding on each other's territories. The company controlling an online platform has no need for video or image capture, it can surveil at the level of abstract data. For example, the game Fortnite has extensive replay capabilities, but this is not a recorded video. Following the early days of machinima when Doom demo movies were shared over low bandwidth connections as logs of keystrokes (Lowood 2006), Fortnite records all server network traffic and player actions for a given game, which can then be used to re-run the round in its entirety. This not only provides a compressed storage option but also allows for replay cameras to move freely around, finding new perspectives after the fact. Within Ready Player One, however, the link to broader image- and screen-based perceptions of privacy reflects back to existing surveillance laws in physical spaces (such as those governing CCTV). Cline is using the metaphor to create an affective intrusion in order to connect with the reader. The dilemma here is instead the assumption that the OASIS (which could record absolutely everything that happens within it) is a neutral context. While the platform acts as the backdrop to the novel, Cline effects a suspension of its power in the absence of an owner as a means of challenging the existing power structures that underpin the platforms and systems that define our social and digital reality.

It is tempting to see the use of data hungry platforms as entailing a form of complicity on the part of users. While it is true that all users can and should pay better attention to terms and conditions, it is entirely unfair to place the burden on the end user. Whitson (2015: 348) claims that "what is important here is that this is willing self-surveillance" but that a gamified system itself "enrolls people into self-governance by using their highest aspirations and capacities, that of self-care and self-development." Aside from the clear postdisciplinary (Foucault) and even post-desire (Deleuze) manipulation at work in this distributed 
protocological system of power, there always remains a question mark over issues of informed consent and participation. Not only are the rules of the game always opaque and unevenly balanced, but as Ready Player One demonstrates in an extreme example, willingness itself is also questionable. When life online provides such improved access to resources, earning potential, products, and sociality, can we ever really choose to disengage? The broader "game" emerges once again, and blame should not be placed on users if society as a whole is so rigged that we have no choice but to participate.

Examples such as Walkaway paint a rose-tinted view of the ease with which an individual can simply leave mainstream society, particularly amidst the metagame that manufactures consent and participation through behavioural manipulation. In Ready Player One, this is shown when the protagonist gets himself caught in IOI's debt-based "indentured employment," functionally slavery, and is forced into the constant worker surveillance and feedback system using not only third-person office-based surveillance but first-person headset cameras that convert the human employee into an avatar, a character in the game of work. As Wade describes, "the voice drove me nuts at first, but I gradually got used to it. I didn't have much choice" (Cline 2012: 280). The characters of this narrative cannot simply "leave" the broader game of society for the online world or any other alternative, and Wade has to purposefully enter this enslavement for a time in order to subvert the corporate surveillance mechanisms and steal information that will enable him and his collaborators to overcome the power structures, evening out the power distribution in order to make playing at society a fairer game. In Ready Player One, we have a happy ending where Wade defeats the corporate enemy and keeps the OASIS as an open and inclusive space. But can we really turn surveillance back on itself? Can anyone play at control? Should power in society be a gamified achievement to be won?

\section{CTRL \{reappropriation: fiction as countergamification\}}

How can society fight back against gamified surveillance in practice? Is there any alternative when our participation is forced upon us by employers, social pressures, and other citizens as the price of entry to a potentially improved situation? One proposed method is counterplay, using gamified systems for alternative purposes. Whitson $(2015: 349,355)$ suggests that gamification can create "critical-reflective heterotopic spaces," but it can also create dystopic spaces. She points out that "the key to maintaining knife-edge heterotopias is to allow gamer subjectivities and playful acts to remake everyday space, without imposing top-down corporate surveillance and governance measures." This is achieved by players making up their own collective games, but this still risks handing data over to corporate and state interests, and Whitson herself had previously written that "there is no place in these systems for the mutual negotiation and agreement upon the rules" (Whitson 2013: 175).

As a social contract, these systems are highly authoritarian, and often foisted upon us. There is instead a need to collectively reshape the broader rules of society in which gamification and surveillance are implicated. While the novels discussed here inevitably fail to provide a complete solution to the problem of playing control in society, they offer small examples of counterplay as an alternative. For example, in Walkaway we see the gaming of situations where the walkaways fly their network drones close to default surveillance drones, so that when anti-drone weapons target the network they also remove the surveillance apparatus (Doctorow 2017). However, in the connected web of power structures and existing frameworks we must be wary of counter-countergame or processes of counter-countersurveillance. For example, even the fact of using encryption or VPNs (virtual private networks) can raise red flags for surveillance mechanisms, as it suggests a user has "something to hide." The texts presented here demonstrate the constant escalation of game-countergame and surveillance-countersurveillance, such as the appropriation of the personal data "cleaner" in "Scroogled" to protect the reputation of selected politicians, the uneven distribution and weaponization of transparency in The Circle, or the technological and literal battles between "default" and the walkaways in Walkaway. It is likely that overcoming gamified surveillance that enables control and protocological power structures will require changing the rules themselves. The difficulty here lies in finding ways to disrupt the smooth running of participation and consent manufacture that perpetuates the existing system. 
Gamification increases participation in surveillance and control systems. Gamifying society introduces an immersive quality to broader power games, a suspension of disbelief that focuses users on the scores and rules of the system. Videogames "allow players to act 'as if' their choices were not constrained by the rules" (Whitson and Simon 2014: 310). On one hand, this function of games enhances participation and compliance in the data gathering and feedback loops of control-based society. But on the other hand, the same ability to play with control acts as a subversive societal game for exploring alternative arrangements of power, enabling speculative gestures towards systems of control. These two sides are often indistinguishable in their methods, blurred in their motives, and mutual in the escalation of their own power game. In this article, we have used fiction as a staging of these games as thought experiments, extrapolating their broader social implications in order to "play" with the rules of society. O'Donnell (2014) warns against treating everything in society as a game, as it loses the "willingness to participate" element of playing a game. But gamification has already displaced this conception of gaming. When gamification is enforced by employers and states, the optional element of play has already been removed: we are being played and played with, our agency displaced, and therein lies the individual and social horror. As Andrew (2015: 353) suggests, if giving up privacy is a requirement of using a particular app or service (especially one with social pressures for participation), it is therefore not voluntary. Entering digital society becomes a Faustian compact (Zuboff 2019: 238), but where Zuboff sees a shift in the application of these logics from digital to physical to social, this article has argued that gamification and its application through surveillance has always been social, relational, and embedded beneath broader networks of power.

The reduction of games to their functional aspect in gamification echoes the increasingly metrics-based approach to society in politics, finance, education, health, and other areas, and Bogost (2015) suggests that as an exercise in exploitation and manipulation, gamification will soon be "stained the same brown hue" as marketing, business, and politics. The gamified system itself is always wrapped in a network of social forces, influences, and obligations. For example, in Second Life normative behaviours are encouraged through social connections rather than any enactment of the possible total surveillance structure of the virtual environment (Wang, Haines, and Tucker 2011). Echoing Doctorow's constant drive towards open technology and creative commons, Chun (2016: 172) suggests that we should move towards thinking in terms of public rights, ephemerality, and being in public (exposed) without being exploited. Escaping the power structures and enforced participation of gamified surveillance requires us to reappropriate society within fiction, to think and write alternative systems of power and its use of technology.

\section{DELETE? \{conclusion\}}

Writing surveillance in and for a gamified society entails creating fiction, games, and other media that generate such alternative social narratives with which users can collectively shift the rules of the game, evening the distribution of power, and disrupting the games of control that define our digital age. The authors discussed take different approaches to gamified power structures that create and use enclosures of total surveillance. Cline focuses on corporate power and the games between companies, Eggers explores the potential conversion of state to corporate power, while Stross and Doctorow adopt a more nuanced approach to the issues arising in the cracks that emerge through the conflation of state and private power. Eggers takes a pessimistic view towards the escalation of tech power games, Cline more positively suggests that the underdog can win the game, Stross focuses on the bewilderment of individuals caught in these games, and Doctorow highlights the risks and radical potential for gamification and surveillance as control and countercontrol. But it is the literary process itself, the expansion of power structures into full social realities that occurs through these fictional thought experiments, that allows us to view the shift in scale between types of surveillance and control games. Echoing Boellstorff's levels of game studies, we have demonstrated individuals being caught in specific gamified systems of control, the spread of this control across specific systems, and the "levelling up" of power games to corporations and states. In each increase of level, the previous level becomes the new resource that is used to keep score. At the highest level of surveillance society, the game is played using citizens as non-player characters and gamification as the means of controlling the global gamified environment. Writing (about) surveillance and gamified control can warn us 
of emerging power structures, critique sociotechnical relations, and offer a space in which new cultures can emerge. All these processes are necessary for the creation of new collective voices that can counter the increasing trend towards gamified society through mass surveillance.

\section{README \{references\}}

Andrejevic, Mark. 2009. Privacy, Exploitation, and the Digital Enclosure. Amsterdam Law Forum 1 (4): 47-62.

Andrew, Lori. 2015. Privacy and Data Collection in the Gameful World. In The Gameful World: Approaches, Issues, Applications, edited by Steffen P. Walz and Sebastian Deterding, 359-69. Cambridge, MA: MIT Press.

Atwood, Margaret. 2013. When Privacy Is Theft. New York Review of Books, November $21,2013$. https://www.nybooks.com/articles/2013/11/21/eggers-circle-when-privacy-is-theft/ [accessed March 13, 2019].

Ball, James. 2013. Xbox Live Among Game Services Targeted by US and UK Spy Agencies. Guardian, December 9, 2013. https:/www.theguardian.com/world/2013/dec/09/nsa-spies-online-games-world-warcraft-second-life [accessed March 13, 2019].

Benjamin, Garfield. 2016. The Cyborg Subject: Reality Consciousness Parallax. Basingstoke: Palgrave Macmillan.

Boellstorff, Tom. 2006. A Ludicrous Discipline? Ethnography and Game Studies. Games and Culture 1 (1): $29-35$.

Bogost, Ian. 2015. Why Gamification Is Bullshit. In The Gameful World: Approaches, Issues, Applications, edited by Steffen P. Walz and Sebastian Deterding, 65-79. Cambridge, MA: MIT Press.

Brooker, Charlie. 2018. Black Mirror: Bandersnatch. Directed by David Slade. Film, 90 minutes. United Kingdom: Netflix.

Chun, Wendy. 2016. Updating to Remain the Same: Habitual New Media. Cambridge, MA: MIT Press.

Clarke, Roger. 1988. Information Technology and Dataveillance. Communications of the ACM 31 (5): $498-512$.

Cline, Ernest. 2012. Ready Player One. London: Arrow.

Consalvo, Mia. 2007. Cheating: Gaining Advantage in Videogames. Cambridge, MA: MIT Press.

Crogan, Patrick. 2011. Gameplay Mode: War, Simulation, and Technoculture. Minneapolis: University of Minnesota Press.

Cybulski, Alex Dean. 2014. Enclosures at Play: Surveillance in the Code and Culture of Videogames. Surveillance \& Society 12 (3): 427-32.

Deleuze, Gilles. 1992. Postscript on the Societies of Control. October 59: 3-7.

Deterding, Sebastian, Dan Dixon, Rilla Khaled, and Lennart Nacke. 2011. From Game Design Elements to Gamefulness: Defining "Gamification." MindTrek'11 ACM, September 28-30, 2011, 9-15.

Doctorow, Cory. 2003. Down and Out in the Magic Kingdom. London: HarperCollins.

Doctorow, Cory. 2015. Scroogled. In Pwning Tomorrow: An Anthology of Short Fiction from the Electronic Frontier, edited by Electronic Frontier Foundation, 2223-445. San Francisco, CA: Electronic Frontier Foundation. First published 2007.

Doctorow, Cory. 2017. Walkaway. London: Head of Zeus.

Dragona, Daphne. 2014. Counter-gamification: Emerging Tactics and Practices Against the Rule of Numbers. In Rethinking Gamification, edited by Mathias Fuchs, Sonia Fizek, Paolo Ruffino, and Niklas Schrape, 227-50. Lüneburg, DE: Meson Press.

Eggers, Dave. 2013. The Circle. London: Penguin.

Fang, Lee. 2019. Google Hired Gig Economy Workers to Improve Artificial Intelligence in Controversial Drone-Targeting Project. Intercept, February 4, 2019. https://theintercept.com/2019/02/04/google-ai-project-maven-figure-eight/ [accessed March 13, 2019].

Galloway, Alexander. 2004. Protocol: How Control Exists After Decentralization. Cambridge, MA: MIT Press.

Galloway, Alexander, and Eugene Thacker. 2007. The Exploit: A Theory of Networks. Minneapolis: University of Minnesota Press.

Gibson, William. 1984. Neuromancer. New York, NY: Ace.

Haskins, Caroline. 2019. Amazon's Home Security Company Is Turning Everyone Into Cops. Motherboard, February $7,2019$. https://motherboard.vice.com/en_us/article/qvyvzd/amazons-home-security-company-is-turning-everyone-into-cops [accessed March 13, 2019].

Kuo, Lilly. 2019. China Bans 23M from Buying Travel Tickets as Part of 'Social Credit' System. Guardian, March 1, 2019. https://www.theguardian.com/world/2019/mar/01/china-bans-23m-discredited-citizens-from-buying-travel-tickets-socialcredit-system [accessed March 13, 2019].

"Lowood, Henry. 2006. High-performance play: The making of machinima. Journal of Media Practice 7(1): 25-42.

Netflix. 2018. 2018 Fourth Quarter Earnings. Letter to Shareholders, January 17 , 2019. https://s22.q4cdn.com/959853165/files/doc_financials/quarterly_reports/2018/q4/01/FINAL-Q4-18-Shareholder-Letter.pdf [accessed March 13, 2019].

O’Donnell, Casey. 2014. Getting Played: Gamification, Bullshit, and the Rise of Algorithmic Surveillance. Surveillance \& Society 12 (3): $349-59$.

Oravec, Jo Ann. 2015. Gamification and Multigamification in the Workplace: Expanding the Ludic Dimensions of Work and Challenging the Work/Play Dichotomy. Cyberpsychology: Journal of Psychosocial Research on Cyberspace 9 (3): 1-13.

Schafer, Burkhart. 2013. Crowdsourcing and Cloudsourcing CCTV Surveillance. Datenschutz und Datensicherheit 37 (7): $434-39$.

Schrape, Niklas. 2014. Gamification and Governmentality. In Rethinking Gamification, edited by Mathias Fuchs, Sonia Fizek, Paolo Ruffino, and Niklas Schrape, 21-45. Lüneburg, DE: Meson Press.

Srnicek, Nick. 2017. Platform Capitalism. Cambridge, UK: Polity.

Statista. 2019. Forecast of Video Games Users by Segment in the United States from 2017 to 2023 (in Million). Statista https://www.statista.com/statistics/460129/video-games-users-in-the-united-states-forecast/ [accessed March 13, 2019 ]. 
Stephenson, Neal. 2011. Reamde. London: Atlantic.

Stross, Charles. 2008. Halting State. London: Hachette Digital.

Stross, Charles. 2013. PSA: Why There Won't Be a Third Book in the Halting State Trilogy. Charlie's Diary [blog], December 9, 2013. http://www.antipope.org/charlie/blog-static/2013/12/psa-why-there-wont-be-a-third-.html [accessed March 13, 2019].

Wang, Victoria, Kevin Haines, and John Tucker. 2011. Deviance and Control in Communities with Perfect Surveillance: The Case of Second Life. Surveillance \& Society 9 (1/2): 31-46.

Whitson, Jennifer. 2013. Gaming the Quantified Self. Surveillance \& Society 11(1/2): 163-76.

Whitson, Jennifer. 2015. Foucault's Fitbit: Governance and Gamification. In Gameful Worlds: Approaches, Issues, Applications, edited by Steffen P Walz and Sebastian Deterding, 339-58. Cambridge, MA: MIT Press.

Whitson, Jennifer, and Bart Simon. 2014. Game Studies Meets Surveillance Studies at the Edge of Digital Culture: An Introduction to a Special Issue on Surveillance, Games and Play. Surveillance \& Society 12(3): 309-19.

Zuboff, Shoshana. 2019. The Age of Surveillance Capitalism: The Fight for a Human Future at the New Frontier of Power. New York, NY: Hachette. 